Pure and Applied Mathematics Quarterly

Volume 6, Number 3

(Special Issue: In honor of

Joseph J. Kohn, Part 1 of 2)

$755-780,2010$

\title{
Inclusions between Parabolic Geometries
}

\author{
Boris Doubrov and Jan Slovák \\ Dedicated to Professor J.J.Kohn on the occasion of his 75th birthday
}

\begin{abstract}
Some of the well known Fefferman like constructions of parabolic geometries end up with a new structure on the same manifold. In this paper, we classify all such cases with the help of the classical Onishchik's lists [10] and we treat the only new series of inclusions in detail, providing the spinorial structures on the manifolds with generic free distributions. Our technique relies on the cohomological understanding of the canonical normal Cartan connections for parabolic geometries and the classical computations with exterior forms. Apart of the complete discussion of the distributions from the geometrical point of view and the new functorial construction of the inclusion into the spinorial geometry, we also discuss the normality problem of the resulting spinorial connections. In particular, there is a non-trivial subclass of distributions providing normal spinorial connections directly by the construction.
\end{abstract}

Keywords: Cartan connections, Fefferman construction, free distributions, spinorial geometry, normality conditions.

\section{INTRODUCTION}

This paper is motivated by two recent examples of conformal structures naturally associated with non-degenerate rank 2 vector distributions on 5-dimensional

Received July 22, 2008.

Research supported by the Eduard Čech Center for Algebra and Geometry and the grant GACR 201/08/0397. 
manifolds [9] and with non-degenerate rank 3 distributions on 6-dimensional manifolds [2]. In both cases there are natural parabolic geometries associated with these distributions, which serve as an intermediate structure between the distribution and the conformal geometry.

Example 1 (Nurowski [9]). Let $D$ be a rank 2 non-degenerate vector distribution on 5-dimensional manifold $M$. The non-degeneracy condition means that the derived spaces $D^{2}=D+[D, D]$ and $D^{3}=D^{2}+\left[D, D^{2}\right]$ have the maximal possible dimensions 3 and 5 respectively. Elie Cartan proved in his famous paper [6], that there is a natural $G_{2}$-geometry associated with any such distribution. Pavel Nurowski noticed that this geometry can be extended into the conformal geometry of signature $(3,2)$ using the classical embedding of the split real form of $G_{2}$ into $\mathfrak{s o}(4,3)$. Thus, there exists a natural cone of null-vectors $\mathcal{C} \subset T M$ associated with any such distribution.

Example 2 (Bryant [2]). Similarly, let $D$ be a rank 3 vector distribution on a 6-dimensional manifold $M$. We assume that $D$ is non-degenerate, that is $D^{2}=$ $D+[D, D]$ coincides with all $T M$. Robert Bryant [2] showed that there is a natural $S O(4,3)$-geometry associated with each such distribution and then used the spinor representation $\mathfrak{s o}(4,3) \rightarrow \mathfrak{s o}(4,4)$ in order to extend it to the conformal geometry of type $(3,3)$ on the manifold $M$. In particular, there is also a natural cone of null-vectors $\mathcal{C} \subset T M$ associated with any such distribution.

Both examples have striking similarity. Firstly, they both start with nondegenerate vector distributions, which generate parabolic geometries. All such distributions were described by K. Yamaguchi [15] using the theory of geometric structures associated with filtered manifolds [13]. Secondly, the associated parabolic geometries can be embedded into another parabolic geometry, the conformal one.

This raises the natural question: What are all possible embeddings of one parabolic geometry into another, and what geometric meanings do they have? We answer these questions, using the classical Onishchik's list [10] of all possible inclusions between complex parabolic homogeneous spaces. It appears that there are only three such examples (two of them are series of geometries in appropriate dimensions). One of the series provides the trivial inclusion of the contact projective geometry (cf. D. Fox, [7]) into the projective geometry. The second example is the embedding of $G_{2}$ geometry into the conformal $(3,2)$ geometry 
used by P. Nurowski. Finally, the third example produces the embedding of $B_{l^{-}}$ geometry into $D_{l+1}$-geometry, which was used in the smallest dimension $l=3$ by R. Bryant.

We explore the latter series for an arbitrary $l$ in more detail. As a result, we show that any non-degenerate $l$-dimensional vector distribution on $l(l+1) / 2$ dimensional manifold $M$ induces a natural almost spinorial structure on $M$ associated with it. An almost spinorial structure on a manifold $M$ is given by an isomorphism of the tangent bundle $T M$ with the vector bundle $\Lambda^{2} S$ for some vector bundle $S$ over $M$. This structure can be considered as a way of identifying each tangent space $T_{p} M, p \in M$, with the set of skew-symmetric matrices with $(l+1)$ rows and columns. In particular, for odd $l$ each such structure defines a cone $\mathcal{C}$ in $T M$ consisting of all degenerate skew-symmetric matrices, which are given as zeros of the Pfaffian. For example, in the case of $l=3$ which was considered by R. Bryant, we get the quadratic non-degenerate cone. So, in this smallest possible dimension the almost spinorial structure coincides with the conformal structure of signature $(3,3)$.

After providing a very brief review of the main concepts of parabolic geometries, the inclusions are studied and classified for the homogeneous models. This leads to the complete classification in Theorem 2 and a full understanding of the simple construction of the spinorial geometry for the distributions. At the same time this raises many natural questions about the relations between the two geometries. Quite straightforward computations exploiting the understanding of the normal Cartan connections lead to detailed description of the fundamental invariants of the geometries in Theorems 3 and 4 . Finally the study of the main geometric objects is continued and the normality questions are discussed in the rest of the article, cf. Theorem 5 and Example 3.

\section{General parabolic geometries}

The parabolic geometries are curved deformations of the homogeneous spaces $G / P$ with $G$ semisimple and $P$ parabolic. Thus, a parabolic geometry of type $(G, P)$ on a manifold $M$ is a principal fiber bundle $\mathcal{G} \rightarrow M$ with structure group $P$, equipped with an absolute parallelism $\omega \in \Omega^{1}(\mathcal{G}, \mathfrak{g})$ which is $\mathrm{Ad}$-invariant with respect to the principal $P$-action and reproduces the fundamental vector fields. 
The form $\omega$ is called the Cartan connection of type $(G, P)$ on $M$. Originally, Cartan built more general absolute parallelisms by means of his famous equivalence method. Nowadays, the Cartan connections appear in many areas of geometric analysis and there is a rich theory introducing various types of calculi and general structural results, see [5] for a detailed treatment.

Most general features of the individual types of the parabolic geometries are read off the algebraic properties of the so called flat models $G \rightarrow G / P$, where $\omega$ is the Maurer-Cartan form. On the other hand, at curved manifolds, the parabolic geometry is rather given by some explicit and simple structure visible at the manifold itself, while $\omega$ is uniquely determined by a construction based on a natural normalization. We have mentioned the well known examples of projective, conformal and spinorial geometries above.

The crucial algebraic structures are derived from the grading $\mathfrak{g}=\mathfrak{g}_{-k} \oplus \cdots \oplus \mathfrak{g}_{k}$ of the semisimple Lie algebra $\mathfrak{g}$ giving rise to the parabolic subalgebra $\mathfrak{p}=\mathfrak{g}_{0} \oplus$ $\cdots \oplus \mathfrak{g}_{k}$. At the level of the curved geometries, this yields the $P$-invariant filtration on $T \mathcal{G}$ which projects also to the filtration on $T M$. Let us also notice that the Cartan-Killing form identifies $\mathfrak{p}_{+}=\mathfrak{g}_{1} \oplus \cdots \oplus \mathfrak{g}_{k}$ with $(\mathfrak{g} / \mathfrak{p})^{*}$ as $P$-modules and $\mathfrak{g} / \mathfrak{p}$ equals to $\mathfrak{g}_{-}=\mathfrak{g}_{-k} \oplus \cdots \oplus \mathfrak{g}_{-1}$ as $G_{0}$-module, where $G_{0}$ is the reductive part of the parabolic subgroup $P$ (with Lie algebra $\mathfrak{g}_{0}$ ).

It is also well known, how to understand the structure of the geometries in cohomological terms. The curvature form $\Omega \in \Omega^{2}(\mathcal{G}, \mathfrak{g})$ of the Cartan connection $\omega$ is given by the structure equation

$$
\Omega=d \omega+\frac{1}{2}[\omega, \omega]
$$

and the absolute parallelism allows to express the curvature by the curvature function $\kappa: \mathcal{G} \rightarrow \wedge^{2} \mathfrak{p}_{+} \otimes \mathfrak{g}, \kappa(X, Y)=K\left(\omega^{-1}(X), \omega^{-1}(Y)\right)$. Thus, the curvature function has values in the cochains of the Lie algebra cohomology of $\mathfrak{g}_{-}$with coefficients in $\mathfrak{g}$. This cohomology is explicitly computable by the Kostant's version of the BBW theorem, cf. $[8,12,5]$, and we may compute it either by means of the standard differential $\partial$ or by its adjoint co-differential $\partial^{*}$. The formula in the special case of the above two-chains is

$$
\partial^{*}\left(Z_{0} \wedge Z_{1} \otimes X\right)=-Z_{0} \otimes\left[Z_{1}, X\right]+Z_{1} \otimes\left[Z_{0}, X\right]-\left[Z_{0}, Z_{1}\right] \otimes X .
$$

Another important property of the parabolic geometries imposes conditions on the behavior of the filtrations and is called regularity. In words, the filtrations 
have to respect the Lie brackets of vector fields. In terms of the curvature, this says that no curvature components of non-positive homogeneities are allowed.

The Tanaka theory, further extended and worked out in last thirty years, shows that normalizing the regular Cartan connections properly defines an equivalence of categories of certain filtered manifolds (with additional simple geometric structures under some cohomological conditions, like for all $|1|$-gradings or contact gradings) and categories of Cartan connections, cf. $[13,4,5]$. Then the harmonic part of the curvature defines all the rest and, in particular, the geometry is locally isomorphic to its flat model if and only if the curvature vanishes. Moreover, the entire curvature tensor is computable explicitly by a natural differential operator from its harmonic part, cf. [3].

\section{InClusions Between PARABOliC GeOMETRIES}

Quite often, there are natural constructions linking together different parabolic geometries. For example, the Fefferman's celebrated construction of a conformal structure on a circle bundle over each hypersurface type CR-manifold allows to exploit the much simpler invariant theory of the conformal Riemannian structures in order to understand that of the CR-geometry.

Of course, each such construction is of functorial character and it is determined at the algebraic level already at the homogeneous models. Thus we shall start at the level of Lie groups and we describe the main ingredients of the Fefferman like constructions.

Let $G / P$ and $\tilde{G} / \tilde{P}$ be two (real or complex analytic) parabolic homogeneous spaces, i.e. $G$ is any semisimple Lie group and $P \subset G$ a parabolic subgroup, and consider an homomorphism $i: G \rightarrow \tilde{G}$ which is infinitesimally injective.

Second, we require that the $G$-orbit of $o=e \tilde{P} \in \tilde{G} / \tilde{P}$ is open. This means that the map $\mathfrak{g} \rightarrow \tilde{\mathfrak{g}} / \tilde{\mathfrak{p}}$ induced by $i^{\prime}: \mathfrak{g} \rightarrow \tilde{\mathfrak{g}}$ is surjective.

Now, the subgroup $Q:=i^{-1}(\tilde{P})$ is a closed subgroup of $G$, which is usually not parabolic. The homomorphism $i$ then induces a smooth map $G / Q \rightarrow \tilde{G} / \tilde{P}$, whose image is the $G$-orbit of $o$.

Finally, we need that $P \subset G$ contains $Q$. Having secured all this, there is the natural projection $\pi: G / Q \rightarrow G / P$. The homomorphism $i: G \rightarrow \tilde{G}$ induces the smooth map $G / Q \rightarrow \tilde{G} / \tilde{P}$ which is a covering of the $G$-orbit of $o$, and as an open 
subset in $\tilde{G} / \tilde{P}$ carries a canonical geometry of type $(\tilde{G}, \tilde{P})$. This can be pulled back to obtain such a geometry on $G / Q$.

Of course, if we replace Lie groups $G, \tilde{G}$ and their Maurer-Cartan forms by the principal fiber bundles and Cartan connections, the same construction applies with the Lie subgroups $P, \tilde{P}$ and $Q$, see [5, Section 4.5] for general theory and several examples.

Especially, it may happen that

$$
i(G) \tilde{P}=\tilde{G} \text { and } i(P)=i(G) \cap \tilde{P}
$$

i.e. $Q=P$ is the parabolic subgroup. Then both parabolic geometries turn out to live over the same base manifold $G / P=\tilde{G} / \tilde{P}$. We say that $i$ is an inclusion of parabolic homogeneous spaces. For curved geometries of these types we talk about inclusions of parabolic geometries.

This is equivalent to the conditions that $i(G)$ acts transitively on the manifold $\tilde{G} / \tilde{P}$ and the stationary subgroup of this action at $o=e \tilde{P}$ coincides with $i(P)$.

All such non-trivial inclusions in complex analytic case were described by A. Onishchik [10] (see also [11, §15] for more details). It appears that if $G$ is simple, there is a very limited number of such examples.

Theorem 1 (Onishchik, [10]). Let $M=G / P$ be a complex parabolic homogeneous space. Let $\tilde{G}=(\operatorname{Bih} M)^{\circ}$ be the connected component of the group of all biholomorphic automorphisms of $M$. If $G$ is simple, then $\tilde{G}$ is also simple. Moreover, $\tilde{G}$ always coincides with $G$ with the following exceptions:

(1) $G=P S p(2 l, \mathbb{C}), P=P_{\Sigma}$, where $\Sigma=\left\{\alpha_{2}, \ldots, \alpha_{l}\right\}, M=\mathbb{C P}^{2 l-1}, \tilde{G}=$ $P S L(2 l, \mathbb{C})$;

(2) $G=G_{2}, P=P_{\left\{\alpha_{2}\right\}}, M=Q^{5}, \tilde{G}=P S O(7, \mathbb{C})$;

(3) $G=S O(2 l+1, \mathbb{C}), P=P_{\Sigma}$, where $\Sigma=\left\{\alpha_{1}, \ldots, \alpha_{l-1}\right\}, M=I^{o} \operatorname{Gr}_{l+1}\left(\mathbb{C}^{2 l+2}\right)$, $\tilde{G}=P S O(2 l+2, \mathbb{C})$.

The first exceptional geometries are the complex versions of the so called projective contact structures. The real split form of the symplectic algebra is the only one allowing this complexified parabolic subalgebra, cf. [5, Section 2.3]. The inclusion can be also nicely interpreted with the help of the distinguished geodesics related to the projective geometry and these questions have been studied in great detail by D. Fox, [7]. 
In the second case, $Q^{5}$ denotes the quadric in $\mathbb{C P}^{6}$ given by the equation $(z, z)=0$, where the scalar product on $\mathbb{C}^{7}$ is given by the standard $S O(7, \mathbb{C})$ representation, and $G_{2}$ is embedded into $S O(7, \mathbb{C})$ by its unique (up to the conjugation) irreducible 7-dimensional representation, which has an invariant nondegenerate symmetric form. Thus this corresponds to the Nurowski's example above. Again, the split real form is the only one allowing this complexified parabolic subalgebra.

The space $I^{o} \mathrm{Gr}_{l+1}\left(\mathbb{C}^{2 l+2}\right)$ denotes the connected component of the manifold of isotropic Lagrangian subspaces in $\mathbb{C}^{2 l+2}$, which contains $V_{0}=\left\langle e_{1}, \ldots, e_{l+1}\right\rangle$. Here $\left\{e_{1}, \ldots, e_{2 l+2}\right\}$ is a basis in $\mathbb{C}^{2 l+2}$, such that $S O(2 l+2, \mathbb{C})$-invariant symmetric form has the matrix:

$$
\left(\begin{array}{cc}
0 & E_{l+1} \\
E_{l+1} & 0
\end{array}\right) .
$$

In order to understand better the third example in the list of exceptions, let us work out the explicit description of the algebraic inclusion. Again, only the split real form allows for such parabolics and so we shall deal with these real Lie algebras.

Let us identify $S O(l, l+1)$ with the set of matrices preserving the following symmetric form:

$$
\left(\begin{array}{ccc}
0 & 0 & E_{l} \\
0 & 1 & 0 \\
E_{l} & 0 & 0
\end{array}\right) .
$$

Then the embedding $i: G \rightarrow \tilde{G}$ can be described infinitesimally by the following injective mapping of the corresponding Lie algebras:

$$
\begin{gathered}
\alpha: \mathfrak{s o}(l, l+1) \mapsto \mathfrak{s o}(l+1, l+1), \\
\left(\begin{array}{ccc}
A & X & Y \\
-Z^{t} & 0 & -X^{t} \\
T & Z & -A^{t}
\end{array}\right) \mapsto\left(\begin{array}{cccc}
A & \frac{1}{\sqrt{2}} X & \frac{1}{\sqrt{2}} X & Y \\
-\frac{1}{\sqrt{2}} Z^{t} & 0 & 0 & -\frac{1}{\sqrt{2}} X^{t} \\
-\frac{1}{\sqrt{2}} Z^{t} & 0 & 0 & -\frac{1}{\sqrt{2}} X^{t} \\
T & \frac{1}{\sqrt{2}} Z & \frac{1}{\sqrt{2}} Z & -A^{t}
\end{array}\right)
\end{gathered}
$$

where $A, Y, T \in \operatorname{Mat}_{l}(\mathbb{R}), X, Z \in \mathbb{R}^{l}, Y+Y^{t}=T+T^{t}=0$.

Clearly, the $P$-module structure on $\mathfrak{g}$ reveals that the filtration of a parabolic geometry of type $(G, P)$ is given by the distribution of rank $l$ on a manifold of 
dimension $\frac{1}{2}(l+1) l$. Since the first cohomology $H^{1}\left(\mathfrak{g}_{-}, \mathfrak{g}\right)$ concentrates in negative homogeneities only, the filtration determines the normal parabolic geometry completely, cf. [5, Section 4.3]. Concerning the geometry of type $(\tilde{G}, \tilde{P})$, we have mentioned already that this is one of the examples of geometries with trivial filtration and determined by a classical G-structure, which is given by an identification of $T M$ with the second exterior tensor power of an auxiliary vector bundle $S$ of dimension $l+1$, cf. [5, Section 4.1].

Theorem 2. The only inclusions of real parabolic geometries with simple Lie groups $G$ and $\tilde{G}$ are the following ones:

(1) The obvious projective structure induced by the contact projective geometries (see [7] and [5, Section 4.5]).

(2) The Cartan's example of distributions with grows vectors $(2,3,5)$ on fivedimensional manifolds carrying the conformal Riemannian geometry of signature $(3,2)$ (see $[9])$.

(3) The generic free distribution with grows vector $\left(l, \frac{1}{2}(l+1) l\right), l \geq 3$ carrying the spinorial structures (well known only in dimension $l=3$, [2]).

Proof. If an inclusion of parabolic geometries of given types should exist, then there must be the corresponding inclusion of the homogeneous spaces. However, at the level of the Lie groups everything is realized by real analytic objects. Thus, complexifying, there must exist the appropriate inclusion in the holomorphic category and the Onishchik's list together with the above observations complete the proof.

In the rest of this paper, we shall work out more details on the new series of examples. In order to understand the functorial construction of the spinorial geometry from the rank $l$ distribution, we need a bit more knowledge of both geometries. On the other hand, we shall see that the $G_{0}$-module structure of $\mathfrak{g}_{-}$and the standard representations of $G$ and $\tilde{G}$ are enough to construct quickly the almost spinorial structure on $M$ directly from the Cartan connection $(\mathcal{G}, \omega)$ associated with the rank $l$ distribution $D$ on $M$. The more interesting and difficult questions are:

- How much of the Cartan connection $\omega$ do we need to recover the spinorial geometry? 
- Under which conditions will the induced spinorial Cartan connection $\tilde{\omega}$ be normal again?

We shall come back to these questions in the subsequent sections.

Now, let us consider the associated standard tractor bundle $\mathcal{T} M=\mathcal{G} \times{ }_{P} V$, where $V$ is the standard $S O(l, l+1)$ representation. The action of the parabolic subgroup $P$ preserves the filtration $V=V^{0} \supset V^{1} \supset V^{2} \supset 0$, where $\operatorname{dim} V^{2}=l$, $\operatorname{dim} V^{1}=l+1$. It induces the filtration of the tractor bundle:

$$
\mathcal{T} M=\mathcal{T}^{0} M \supset \mathcal{T}^{1} M \supset \mathcal{T}^{2} M \supset 0
$$

As a $G_{0}$ module, the standard representation splits as $V=\mathbb{R}^{l} \oplus \mathbb{R} \oplus \mathbb{R}^{l}$, where $\mathbb{R}$ is the trivial representation while $\mathbb{R}^{l}=V / V^{1}$ is isomorphic to $\mathfrak{g}_{-1}$. Thus, the $G_{0}$-module

$$
S=V / V^{2}=\mathbb{R} \oplus \mathbb{R}^{l}
$$

has the property $\wedge^{2} S=\mathfrak{g}_{-}$. It is easy to see, that this $G_{0}$ module structure is compatible with the inclusion $G_{0} \rightarrow \tilde{G}_{0}$.

At the level of the parabolic geometry determined by the distribution $D$, we simply consider the auxiliary vector bundle $\mathcal{S}=\mathcal{G} \times{ }_{P} S$ and we see that the tangent bundle $T M$ is naturally isomorphic to $\wedge^{2} \mathcal{S}$. Since the identification is compatible with the inclusion of the reductive parts of the parabolic subgroups, this is the right spinorial structure as obtained from the general construction.

\section{Canonical Cartan connection for length 2 Distributions}

Let us notice, that we have not exploited the entire Cartan connection $\omega$ in the construction above. Rather we have only used the splitting of the $G_{0}$-modules $\mathfrak{g}_{-}$and $V$. Moreover, only the homogeneity one part of the total splitting of $V$ was necessary (we have split $V / V^{2}$ only).

In the Cartan-Tanaka procedure, this amount of information is obtained after the first prolongation step (the bottom up approach). The construction in $[5$, Section 3.1] provides the entire Cartan connection and the complete information on the structure of the curvature, without the explicit prolongation steps. We shall combine these two approaches by using the detailed knowledge on the curvature during the explicit prolongation computations. For the sake of simplicity, we 
shall ignore the lowest dimensional case with $l=3$ since the curvature structure is different and this case is well known.

Let $D$ be a rank $l \geq 4$ vector distribution on a manifold $M$ of dimension $l(l+1) / 2$. We say that $D$ is non-degenerate, if $D+[D, D]=T M$. In other words, if $X_{1}, \ldots, X_{l}$ is any local basis of sections of $D$, then the vector fields $X_{i},\left[X_{j}, X_{k}\right], 1 \leq i \leq l, 1 \leq j<k \leq l$ should form a basis of the tangent space $T M$ at all points where sections $X_{i}$ are defined.

Under this non-degeneracy condition, the general theory implies that there is a natural regular and normal Cartan connection of type $(G, P)$ on $M$, with the corresponding pair of Lie algebras $(\mathfrak{g}, \mathfrak{p})$ given by:

$$
\mathfrak{g}=\left\{\left(\begin{array}{ccc}
A & X & Y \\
-Z^{t} & 0 & -X^{t} \\
T & Z & -A^{t}
\end{array}\right)\right\}, \quad \mathfrak{p}=\left\{\left(\begin{array}{ccc}
A & 0 & 0 \\
-Z^{t} & 0 & 0 \\
T & Z & -A^{t}
\end{array}\right)\right\},
$$

where $A, Y, T \in \operatorname{Mat}_{l}(\mathbb{R}), X, Z \in \mathbb{R}^{l}, Y+Y^{t}=T+T^{t}=0$.

Theorem 3 ([13], [5]). For each non-degenerate distribution of rank $l$ on a manifold of dimension $\frac{1}{2}(l+1) l$, there is the unique regular normal Cartan connection of type $(G, P)$ on $M$ (up to isomorphisms). The only fundamental invariant of these parabolic geometries is concentrated in the homogeneity degree 1 and corresponds to the totally trace-free part of the $\mathfrak{s l}(l, \mathbb{R})$-submodule $\operatorname{Hom}\left(\mathfrak{g}_{-1} \wedge \mathfrak{g}_{-2}, \mathfrak{g}_{-2}\right)$ in the curvature.

Proof. The existence and uniqueness of the Cartan connection follow from the Tanaka theory (see also [5, Section 3.1]), for the explicit computation of the curvature see [5, Section 4.3] or compute following the algorithm of Kostant, see $[8,12]$.

In more detail, let $\pi_{1}, \ldots, \pi_{l-1}$ be the fundamental weights of $\mathfrak{s l}(l, \mathbb{R})$. Then $\mathfrak{g}_{-1}$ has weight $\pi_{1}, \mathfrak{g}_{-2}$ is isomorphic to $\wedge^{2} \mathfrak{g}_{-1}$ and has weight $\pi_{2}$, and by the trace-free part of $\operatorname{Hom}\left(\mathfrak{g}_{-1} \wedge \mathfrak{g}_{-2}, \mathfrak{g}_{-2}\right)$ we mean the unique submodule with the highest weight $\pi_{2}+\pi_{l-2}+\pi_{l-1}$.

Remark 1. Unlike the case of rank 3 distributions, the fundamental invariant for this Cartan connection is a part of the torsion for distributions of rank $l \geq$ 4. In particular, in this case any regular and normal torsion-free geometry is automatically flat, i.e. locally isomorphic to its homogeneous model. 
Now we start our computations. Let $\left\{X_{1}, \ldots, X_{l}\right\}$ be any (local) frame of $D$. Denote then by $X_{[i j]}$ vector fields $-\left[X_{i}, X_{j}\right]$. Then vector fields $\left\{X_{i}, X_{[j k]}\right\}$ will form the frame on $M$. Denote by $\left\{\theta^{i}, \theta^{[j k]}\right\}$ the dual coframe and by $D^{\perp}$ the set of all 1-forms on $M$ annihilating $D$. It is clear that $D^{\perp}$ is generated by $\theta^{[j k]}$.

Note that

$$
d \theta^{[j k]}\left(X_{j}, X_{k}\right)=-\theta^{[j k]}\left(\left[X_{j}, X_{k}\right]\right)=1
$$

This implies that

$$
d \theta^{[j k]}=\theta^{j} \wedge \theta^{k} \bmod \left\langle\theta^{[r s]}\right\rangle .
$$

So, the structure equations of the coframe $\left\{\theta^{i}, \theta^{[j k]}\right\}$ have the form:

$$
\begin{aligned}
d \theta^{r} & =f_{i[j k]}^{r} \theta^{i} \wedge \theta^{[j k]}+f_{[[i j][k l]]}^{r} \theta^{[i j]} \wedge \theta^{[k l]}, \\
d \theta^{[r s]} & =\theta^{r} \wedge \theta^{s}+f_{i[j k]}^{[r s]} \theta^{i} \wedge \theta^{[j k]}+f_{[[i j][k l]]}^{[r s]} \theta^{[i j]} \wedge \theta^{[k l]},
\end{aligned}
$$

where $f_{i[j k]}^{r}, f_{[[i j][k l]]}^{r}, f_{i[j k]}^{[r s]}, f_{[[i j][k l]]}^{[r s]}$ are the structure functions of the coframe $\left\{\theta^{i}, \theta^{[j k]}\right\}$ on $M$ uniquely determined by the choice of the frame $\left\{X_{1}, \ldots, X_{l}\right\}$. Note that these families of functions do not form any tensor, since their transformation rule under the change of the frame involves derivatives. The natural Cartan connection associated with the distribution $D$ will allow us to construct the coframes behaving much nicer and we shall obtain the components of the curvature tensor at the same time.

Let $\pi: \mathcal{G} \rightarrow M$ be any principle $P$-bundle on $M$ and $\omega: T \mathcal{G} \rightarrow \mathfrak{g}$ any regular Cartan connection of type $G / P$. For any section $s: M \rightarrow \mathcal{G}$ we can write explicitly:

$$
s^{*} \omega=\left(\begin{array}{ccc}
\omega_{j}^{i} & \omega^{i} & \omega^{[i j]} \\
-\omega_{j} & 0 & -\omega^{i} \\
\omega_{[i j]} & \omega_{j} & -\omega_{i}^{j}
\end{array}\right)
$$

where $\omega^{[i j]}, \omega_{[i j]}, \omega_{j}^{i}, \omega^{i}, \omega_{j}$ are 1-forms on $M$.

We say that the Cartan connection $(\mathcal{G}, \omega)$ is adapted to the distribution $D$, if $D=\left\langle\omega^{[i j]}\right\rangle^{\perp}$. It is easy to see that this definition does not depend on the choice of the section $s$.

Let $(G, \omega)$ be an adapted Cartan connection. Then we have

$$
D^{\perp}=\left\langle\omega^{[i j]}\right\rangle=\left\langle\theta^{[i j]}\right\rangle,
$$

where, as above, the forms $\theta^{[i j]}$ are defined by fixing a frame $\left\{X_{1}, \ldots, X_{l}\right\}$ on $D$. 
We can always choose such section $s: M \rightarrow \mathcal{G}$ that

$$
\omega^{i}=\theta^{i} \bmod D^{\perp} .
$$

This condition defines $s$ uniquely up to the transformations $s \rightarrow s g$, where $g: M \rightarrow P_{+}$is an arbitrary $P_{+}$-valued gauge transformation.

Consider the component $\Omega^{[i j]}$ of the curvature tensor:

$$
\Omega^{[i j]}=d \omega^{[i j]}-\omega^{i} \wedge \omega^{j}+\omega_{k}^{i} \wedge \omega^{[k j]}-\omega_{k}^{j} \wedge \omega^{[i k]}
$$

(here and below we use the Einstein summation convention).

Let us remind that $\omega$ is regular and so only positive homogeneities may appear in the curvature. This immediately implies that

$$
d \omega^{[i j]}=\omega^{i} \wedge \omega^{j}=\theta^{i} \wedge \theta^{j} \quad \bmod D^{\perp},
$$

and, hence

$$
\omega^{[i j]}=\theta^{[i j]} \quad \text { for all } 1 \leq i<j \leq l .
$$

Compute now the curvature coefficients of degree 1 together with the section normalizations of the form $s \mapsto \tilde{s}=s g$, where $g$ takes values in exp $\mathfrak{g}_{1}$. Any such transformation leads to the following transformation of the pull-back forms $\tilde{s}^{*} \omega$ :

$$
\begin{aligned}
\tilde{\omega}^{[i j]} & =\omega^{[i j]}, \\
\tilde{\omega}^{i} & =\omega^{i}+p_{j} \omega^{[i j]},
\end{aligned}
$$

where functions $p_{j}$ define the mapping $d g: M \rightarrow \mathfrak{g}_{1}$. Assume that

$$
\begin{aligned}
& \omega^{i}=\theta^{i}+C_{[j k]}^{i} \omega^{[j k]}, \\
& \omega_{j}^{i}=A_{k j}^{i} \omega^{k} \bmod D^{\perp}
\end{aligned}
$$

for some functions $A_{k j}^{i}, C_{[j k]}^{i}$. They are transformed by the gauge transformation $g$ according to the following formula:

$$
\begin{gathered}
\tilde{A}_{j k}^{i}=A_{j k}^{i}-\delta_{j}^{i} p_{k} ; \\
\tilde{C}_{[j k]}^{i}=C_{[j k]}^{i}+\delta_{[j}^{i} p_{k]} .
\end{gathered}
$$

We can always find such functions $p_{i}$ that $\sum_{i} A_{i k}^{i}=0$. This determines the section $s$ uniquely up to the transformations $s \mapsto s g$, where $g$ takes values in $\exp \left(\mathfrak{g}_{2}\right)$. 
We have:

$$
\begin{aligned}
\Omega^{[i j]} & =P_{r[s t]}^{[i j]} \omega^{r} \wedge \omega^{[s t]} \quad \bmod \wedge^{2} D^{\perp}, \\
\Omega^{i} & =Q_{[r s]}^{i} \omega^{r} \wedge \omega^{s} \quad \bmod \Lambda^{1}(M) \wedge D^{\perp} .
\end{aligned}
$$

where

$$
\begin{aligned}
P_{r[s t]}^{[i j]} & =f_{r[s t]}^{[i j]}+\delta_{[s}^{[i} A_{r t]}^{j]}+\delta_{r}^{[i} C_{[s t]}^{j]}, \\
Q_{[j k]}^{i} & =C_{[j k]}^{i}+A_{[j k]}^{i} .
\end{aligned}
$$

Assuming that the connection does not have the torsion in the term $\operatorname{Hom}\left(\wedge^{2} \mathfrak{g}_{-1}, \mathfrak{g}_{-1}\right)$ (as it is implied from the normality assumption via Kostant theorem), we get $Q_{[j k]}^{i}=0$, that is

$$
C_{[j k]}^{i}=-A_{[j k]}^{i}
$$

Substituting this equality to the expression of $P_{r[s t]}^{[i j]}$ we get:

$$
P_{r[s t]}^{[i j]}=f_{r[s t]}^{[i j]}+\delta_{[s}^{[i} A_{r t]}^{j]}-\delta_{r}^{[i} A_{[s t]}^{j]} .
$$

Let us remind that $l \geq 4$. It turns out that we can uniquely determine coefficients $A_{j k}^{i}$ by the following two conditions:

- $\sum_{i} A_{i k}^{i}=0$ according to the choice of the section $s$;

- tensor $P_{r[s t]}^{[i j]}$ is totally trace-free.

Indeed, the traces of $A$ 's do not contribute in the right hand side and the tracefree condition on $P$ just determines the rest.

Theorem 4. The trace-free part of the tensor $P_{r[s t]}^{[i j]}$ computed above is the only fundamental invariant of the non-degenerate rank $l$ distribution $D$ on a manifold of dimension $\frac{1}{2}(l+1) l$. Hence, the Cartan connection associated with $D$ is flat if and only if this tensor vanishes identically.

Proof. The statement is a direct consequence of Theorem 3 and the computations above.

In particular, this gives us an explicit condition when an arbitrary non-degenerate distribution $D$ of rank $l \geq 4$ is locally equivalent to the left-invariant distribution on the nilpotent Lie group corresponding to the algebra $\mathfrak{g}_{-}$. 
Remark 2. Let us also comment on the link between the coefficients $A$ and $C$. In general terms, these objects correspond to the choice of partial Weyl connections (the coefficients $A_{j k}^{i}$ ) and the splittings of the filtration (the improvement of the coframe by deforming $\theta^{i}$ ), cf. [5, Chapter 5]. Both of these objects have to be fixed together because they influence the same curvature components in homogeneity one. This has been reflected by the explicit link between $A$ 's and C's.

Having fixed these homogeneity one objects, all the necessary ingredients for the construction of the spinorial geometry are available, see the end of Section 3 above. Still, in general, we cannot replace the normal Cartan connection of the distribution by the simpler construction of the spinorial normal Cartan connection (although regularity is not an issue for one-graded geometry) since we do not know whether the normality will be preserved.

\section{Computation of Coefficients of Degree 2}

Let as continue the computation of the regular normal Cartan connection $\omega$ associated with the distribution $D$ and its curvature tensor $\Omega$. Let us now consider curvature coefficients of degree 2 together with the normalization of the section $s \mapsto s g$, where $g \in \exp \left(\mathfrak{g}_{2}\right)$. Any such transformation leads to the following transformation of the form $\tilde{\omega}$ :

$$
\begin{aligned}
& \tilde{\omega}_{j}^{i}=\omega_{j}^{i}+q_{[i k]} \omega^{[k j]}, \\
& \tilde{\omega}_{i}=\omega_{i}+q_{[i k]} \omega^{k} \quad \bmod D^{\perp} .
\end{aligned}
$$

Assume that

$$
\begin{aligned}
& \omega_{j}^{i}=A_{k j}^{i} \omega^{k}+E_{j[k l]}^{i} \omega^{[k l]}, \\
& \omega_{i}=F_{k i} \omega^{k} \quad \bmod D^{\perp},
\end{aligned}
$$

where the functions $A_{k j}^{i}$ were determined above, and $E_{j[k l]}^{i}, F_{k i}$ are functions to be found. They are transformed by the gauge transformation $g$ by the following formulas:

$$
\begin{aligned}
\tilde{E}_{j[k l]}^{i} & =E_{j[k l]}^{i}+\delta_{k}^{i} q_{[j l]}-\delta_{l}^{i} q_{[j k]}, \\
\tilde{F}_{k i} & =F_{k i}+q_{[k i]} .
\end{aligned}
$$


We see that we can make $F_{k l}$ symmetric by an appropriate choice of $q_{[k l]}$. This determines uniquely the section $s: M \rightarrow \mathcal{G}$.

Let us now proceed with computations of the curvature coefficients of degree 2. We have

$$
\begin{gathered}
\Omega^{[i j]}=d \omega^{[i j]}-\omega^{i} \wedge \omega^{j}+\omega_{k}^{i} \wedge \omega^{[k j]}-\omega_{k}^{j} \omega^{[i k]}= \\
f_{[[k l][r s]]}^{[i j]} \omega^{[k l]} \wedge \omega^{[r s]}+E_{k[r s]}^{i} \omega^{[r s]} \wedge \omega^{[k j]}-E_{k[r s]}^{j} \omega^{[r s]} \wedge \omega^{[i k]}=R_{[[k l][r s]]}^{[i j]} \omega^{[k l]} \wedge \omega^{[r s]},
\end{gathered}
$$

where

$$
R_{[[k l][r s]]}^{[i j]}=f_{[[k l][r s]]}^{[i j]}+\delta_{[l}^{[i} E_{k][r s]}^{j]} .
$$

Next,

$$
\begin{aligned}
& \Omega^{i}=d \omega^{i}+\omega_{k}^{i} \wedge \omega^{k}+\omega^{[i k]} \wedge \omega_{k}= \\
& d\left(\theta^{i}-A_{[j k]}^{i} \omega^{[j k]}\right)+\omega_{k}^{i} \wedge \omega^{k}+\omega^{[i k]} \wedge \omega_{k}=\left(\bmod \Lambda^{2} D^{\perp}\right) \\
& f_{r[s t]}^{i} \omega^{r} \wedge \omega^{[s t]}-\frac{\partial A_{[j k]}^{i} \omega^{l} \wedge \omega^{[j k]}-A_{[j k]}^{i} f_{r[s t]}^{[j k]} \omega^{r} \wedge \omega^{[s t]}+}{\partial \omega^{l}} \\
& E_{k[r s]}^{i} \omega^{[r s]} \wedge \omega^{k}+F_{j k} \omega^{[i k]} \wedge \omega^{j}=S_{j[k l]}^{i} \omega^{j} \wedge \omega^{[k l]},
\end{aligned}
$$

where

$$
S_{j[k l]}^{i}=f_{j[k l]}^{i}-\frac{\partial A_{[k l]}^{i}}{\partial \omega^{j}}-A_{[r s]}^{i} f_{j[k l]}^{[r s]}-E_{j[k l]}^{i}-F_{j[l} \delta_{k]}^{i} \cdot
$$

Finally,

$$
\begin{gathered}
\Omega_{j}^{i}=d \omega_{j}^{i}+\omega_{r}^{i} \wedge \omega_{j}^{r}-\omega^{i} \wedge \omega_{j}+\omega^{[i k]} \wedge \omega_{[k j]}=\left(\bmod D^{\perp}\right) \\
d\left(A_{k j}^{i}\right) \omega^{k}+A_{k j}^{i} d\left(\theta^{k}-A_{r s}^{k} \omega^{[r s]}\right)+E_{j[k l]}^{i} d \omega^{[k l]}+ \\
\left(A_{s r}^{i} \omega^{s}\right) \wedge\left(A_{t j}^{r} \omega^{t}\right)-F_{k j} \omega^{i} \wedge \omega^{k}=T_{j[k l]}^{i} \omega^{k} \wedge \omega^{l},
\end{gathered}
$$

where

$$
T_{j[k l]}^{i}=-\frac{\partial A_{[k j]}^{i}}{\partial \omega^{l}}-A_{r j}^{i} A_{[k l]}^{r}+A_{[k r}^{i} A_{l] j}^{r}+E_{j[k l]}^{i}-\delta_{[k}^{i} F_{l] j}
$$

Let us now compute normalization conditions on coefficients $R, S, T$ that are implied by the normality condition. In order to do this we explicitly compute $\partial^{*}$ for each of these tensors. 
Fix a basis in $\mathfrak{g}: \mathfrak{g}_{-2}=\left\langle E_{[i j]}\right\rangle, \mathfrak{g}_{-1}=\left\langle E_{i}\right\rangle, \mathfrak{g}_{0}=\left\langle E_{j}^{i}\right\rangle, \mathfrak{g}_{1}=\left\langle E^{i}\right\rangle, \mathfrak{g}_{2}=\left\langle E^{[i j]}\right\rangle$. Let us note that Killing form $B$ on $\mathfrak{g}$ is given by $\operatorname{tr} X Y, X, Y \in \mathfrak{g}$, and, in particular, we have:

$$
\begin{aligned}
B\left(E_{[i j]}, E^{[i j]}\right) & =-2 ; \\
B\left(E_{i}, E^{i}\right) & =-2 ; \\
B\left(E_{j}^{i}, E_{i}^{j}\right) & =2 .
\end{aligned}
$$

For simplicity we can always multiply $B$ by $-1 / 2$ and assume that the dual elements to $E_{i}$ and $E_{[j k]}$ are equal to $E^{i}$ and $E^{[j k]}$ respectively.

Using the identification $\mathfrak{g}_{-}^{*}$ with $\mathfrak{p}_{+}$, we can write the tensor $R \in \operatorname{Hom}\left(\wedge^{2} \mathfrak{g}_{-2}, \mathfrak{g}_{-2}\right)$ as an element of $\wedge^{2} \mathfrak{g}_{2} \otimes \mathfrak{g}_{-2}$ :

$$
R=R_{[[k l][r s]]}^{[i j]} E^{[k l]} \wedge E^{[r s]} \otimes E_{[i j]} .
$$

Then we have:

$$
\begin{aligned}
\partial^{*} R=R_{[[k l][r s]]}^{[i j]}\left(E^{[k l]} \otimes\left[E^{[r s]}, E_{[i j]}\right]-E^{[r s]} \otimes\left[E^{[k l]}, E_{[i j]}\right]\right) & = \\
& \sum_{j} R_{[[k j][r s]]}^{[i j]} E^{[r s]} \otimes E_{i}^{k} .
\end{aligned}
$$

So, we see that $\partial^{*} R$ lies in $\mathfrak{g}_{2} \otimes \mathfrak{g}_{0}$ and can be symbolically written as $\partial^{*} R=\operatorname{tr} R$.

Similarly, we have

$$
S=S_{j[k l]}^{i} E^{j} \wedge E^{[k l]} \otimes E_{i}
$$

and

$$
\begin{aligned}
\partial^{*} S=S_{j[k l]}^{i}\left(E^{j} \otimes\left[E^{[k l]}, E_{i}\right]-E^{[k l]} \otimes\left[E^{j}, E_{i}\right]\right) & = \\
& \sum_{i} S_{j[i l]}^{i} E^{j} \otimes E^{l}-S_{j[k l]}^{i} E^{[k l]} \otimes E_{i}^{j} .
\end{aligned}
$$

Thus, we see that $\partial^{*} S$ lies in $\mathfrak{g}_{1} \otimes \mathfrak{g}_{1}+\mathfrak{g}_{2} \otimes \mathfrak{g}_{0}$ and can be symbolically written as $\partial^{*} S=\operatorname{tr} S-S$.

Finally, we have

$$
T=T_{j[k l]}^{i} E^{k} \wedge E^{l} \otimes E_{i}^{j}
$$


and

$$
\begin{gathered}
\partial^{*} T=T_{j[k l]}^{i}\left(E^{k} \otimes\left[E^{l}, E_{i}^{j}\right)-E^{l} \otimes\left[E^{k}, E_{i}^{j}\right]-E^{[k l]} \otimes E_{i}^{j}\right)= \\
\sum_{i} T_{j[i l]}^{i} E^{j} \otimes E^{l}-T_{j[k l]}^{i} E^{[k l]} \otimes E_{i}^{j} .
\end{gathered}
$$

Consequently, $\partial^{*} T$ lies in $\mathfrak{g}_{1} \otimes \mathfrak{g}_{1}+\mathfrak{g}_{2} \otimes \mathfrak{g}_{0}$ and can be symbolically written as $\partial^{*} T=\operatorname{tr} T-T$.

Summarizing, we get

Lemma 1. Let $\kappa_{2}$ be the degree 2 part of the curvature of the regular and normal $B_{l}$ geometry associated with a non-degenerate distribution $D$. Then it can be expressed as a sum of three tensors:

$$
\begin{aligned}
R & \in \operatorname{Hom}\left(\mathfrak{g}_{-2} \wedge \mathfrak{g}_{-2}, \mathfrak{g}_{-2}\right), \\
S & \in \operatorname{Hom}\left(\mathfrak{g}_{-1} \otimes \mathfrak{g}_{-2}, \mathfrak{g}_{-1}\right), \\
T & \in \operatorname{Hom}\left(\mathfrak{g}_{-1} \wedge \mathfrak{g}_{-1}, \mathfrak{g}_{-1}\right) .
\end{aligned}
$$

They satisfy the normality conditions:

$$
\begin{gathered}
\operatorname{tr} R-S-T=0 \\
\operatorname{tr} S+\operatorname{tr} T=0
\end{gathered}
$$

Remark 3 . These normality conditions determine both coefficients $E_{j[k l]}^{i}$ and $F_{i j}$ uniquely assuming that $F$ has been normalized to be symmetric by the appropriate choice of the section $s: M \rightarrow \mathcal{G}$. Indeed, according to (7) and (8), the condition $\operatorname{tr} S+\operatorname{tr} T=0$ reduces to a linear equation on the symmetric part of $F_{i j}$ :

$$
\operatorname{tr}\left(\delta_{[k}^{i} F_{l] j}+F_{j[l} \delta_{k]}^{i}\right)=(1-l)\left(F_{j k}+F_{k j}\right)=\ldots
$$

where the right-hand side depends only on the known terms. This equation determines the symmetric part of $F$ uniquely. Similarly, the condition $\operatorname{tr} R-S-$ $T=0$ determines $E_{j[k l]}^{i}$ in a unique way.

Let us also comment on the geometric contents of the computed coefficients $E$ and $F$. While the $E_{j[k l]}^{i}$ have completed the definition of the Weyl connection (describing the differentiation in the $\mathfrak{g}_{-2}$-directions as given by the splitting fixed by the coefficients $C$, whereas $A_{j k}^{i}$ already fixed the differentials in the $\mathfrak{g}_{-1^{-}}$ directions), the symmetric coefficients $F_{i j}$ represent the homogeneity two part of the Rho tensor. 


\section{Normality obstructions}

Let us compute when our inclusion maps normal $B_{l}$-geometry to a normal $D_{l+1}$-geometry. The map $\alpha: \mathfrak{g} \rightarrow \tilde{\mathfrak{g}}$ induces an isomorphism:

$$
\bar{\alpha}: \mathfrak{g} / \mathfrak{p} \rightarrow \tilde{\mathfrak{g}} / \tilde{\mathfrak{p}}
$$

On the other hand, we can identify $\mathfrak{g} / \mathfrak{p}$ and $\tilde{\mathfrak{g}} / \tilde{\mathfrak{p}}$ with $\mathfrak{p}_{+}$and $\tilde{\mathfrak{p}}_{+}$respectively using Killing forms on $\mathfrak{g}$ and $\tilde{\mathfrak{g}}$ respectively. This immediately implies that the induced isomorphism (of vector spaces) $\phi: \mathfrak{p}_{+} \rightarrow \tilde{\mathfrak{p}}_{+}$has the form:

$$
\phi: \mathfrak{p}_{+} \rightarrow \tilde{\mathfrak{p}}_{+}, \quad\left(\begin{array}{ccc}
0 & 0 & 0 \\
-Z^{t} & 0 & 0 \\
T & Z & 0
\end{array}\right) \mapsto\left(\begin{array}{cccc}
0 & 0 & 0 & 0 \\
0 & 0 & 0 & 0 \\
-\sqrt{2} Z^{t} & 0 & 0 & 0 \\
T & \sqrt{2} Z & 0 & 0
\end{array}\right)
$$

We can fix a similar basis $\tilde{E}^{[i j]}, \tilde{E}_{j}^{i}, \tilde{E}_{[i j]}$ in $\tilde{\mathfrak{g}}$, where the indices run now from 0 to $l$, in such a way that

$$
\begin{aligned}
\phi\left(E^{[k l]}\right) & =\tilde{E}^{[k l]}, \\
\phi\left(E^{l}\right) & =\sqrt{2} \tilde{E}^{[0 l]}, \\
\alpha\left(E_{[i j]}\right. & =\tilde{E}_{[i j]}, \\
\alpha\left(E_{i}\right) & =\frac{1}{\sqrt{2}}\left(\tilde{E}_{i}^{0}+\tilde{E}_{[0 i]}\right), \\
\alpha\left(E_{j}^{i}\right) & =\tilde{E}_{j}^{i} .
\end{aligned}
$$

The problem of mapping normal $B_{l}$-connections to normal $D_{l+1^{-} \text {-connections }}$ can be reformulated as follows. Let $\kappa \in \wedge^{2} \mathfrak{p}_{+} \otimes \mathfrak{g}$ be the structure function of the normal $B_{l}$-connection., i.e., it satisfies the equation $\partial^{*} \kappa=0$. The mapping $\phi$ can be naturally extended to the morphism $\wedge^{k} \mathfrak{p}_{+} \otimes \mathfrak{g} \rightarrow \wedge^{k} \tilde{\mathfrak{p}}_{+} \otimes \tilde{\mathfrak{g}}$, which is defined as the natural extension of $\phi$ on $\wedge^{k} \mathfrak{p}_{+}$and as the embedding $\alpha$ on $\mathfrak{g}$.

The normality question can be stated as follows: Under which conditions is the element $\phi(\kappa) \in \wedge^{2} \tilde{\mathfrak{p}}_{+} \otimes \tilde{\mathfrak{g}}$ co-closed too?

Let us introduce the operator $\left[\partial^{*}, \phi\right]=\partial^{*} \phi-\phi \partial^{*}$. It acts from $\wedge^{2} \mathfrak{p}_{+} \otimes \mathfrak{g}$ to $\wedge^{2} \tilde{\mathfrak{p}}_{+} \otimes \tilde{\mathfrak{g}}$. As $\kappa$ itself is coclosed, it is clear that $\phi(\kappa)$ is coclosed if and only if $\left[\partial^{*}, \phi\right](\kappa)=0$. 
Define the following elements in $\tilde{\mathfrak{g}}$ :

$$
\begin{aligned}
& \Delta \tilde{E}^{i}=\tilde{E}^{[0 i]}-\tilde{E}_{0}^{i}, \\
& \Delta \tilde{E}_{i}=\tilde{E}_{[0 i]}-\tilde{E}_{i}^{0} .
\end{aligned}
$$

We shall also need the following commutation relations that are easy to compute:

$$
\begin{aligned}
{\left[\Delta \tilde{E}^{i}, \alpha\left(E_{s}^{r}\right)\right] } & =-\delta_{s}^{i} \Delta \tilde{E}^{s} ; \\
{\left[\Delta \tilde{E}^{i}, \alpha\left(E_{s}\right)\right] } & =-\delta_{s}^{i} \Delta \tilde{E}_{0}^{0} ; \\
{\left[\Delta \tilde{E}^{i}, \alpha\left(E_{[r s]}\right)\right] } & =\delta_{[r}^{i} \Delta \tilde{E}_{s]} ; \\
{\left[\Delta \tilde{E}^{i}, \alpha\left(E^{r}\right)\right] } & =0 ; \\
{\left[\Delta \tilde{E}^{i}, \alpha\left(E^{[r s]}\right)\right] } & =0 .
\end{aligned}
$$

Let us compute the operator $\left[\partial^{*}, \phi\right]$ explicitly. For example, for any $\kappa=$ $E^{[i j]} \wedge E^{[k l]} \otimes X, X \in \mathfrak{g}$ we have:

$$
\begin{aligned}
\phi(\kappa) & =\tilde{E}^{[i j]} \wedge \tilde{E}^{[k l]} \otimes \alpha(X) ; \\
\partial^{*} \phi(\kappa) & =\tilde{E}^{[i j]} \otimes\left[\tilde{E}^{[k l]}, \alpha(X)\right]-\tilde{E}^{[k l]} \otimes\left[\tilde{E}^{[i j]}, \alpha(X)\right] ; \\
\partial^{*}(\kappa) & =E^{[i j]} \otimes\left[E^{[k l]}, X\right]-E^{[k l]} \otimes\left[E^{[i j]}, X\right] ; \\
\phi \partial^{*}(\kappa) & =\tilde{E}^{[i j]} \otimes \alpha\left(\left[E^{[k l]}, X\right]\right)-\tilde{E}^{[k l]} \otimes \alpha\left(\left[E^{[i j]}, X\right]\right)= \\
& =\tilde{E}^{[i j]} \otimes\left[\tilde{E}^{[k l]}, \alpha(X)\right]-\tilde{E}^{[k l]} \otimes\left[\tilde{E}^{[i j]}, \alpha(X)\right] .
\end{aligned}
$$

Here we use the fact that $\alpha$ is a homomorphism of Lie algebras. We get:

$$
\left[\partial^{*}, \phi\right]: E^{[i j]} \wedge E^{[k l]} \otimes X \mapsto 0 .
$$


Similarly, for $\kappa=E^{i} \wedge E^{[j k]} \otimes X, X \in \mathfrak{g}$ we have:

$$
\begin{aligned}
\phi(\kappa) & =\sqrt{2} \tilde{E}^{[0 i]} \wedge \tilde{E}^{[j k]} \otimes \alpha(X) ; \\
\partial^{*} \phi(\kappa) & =\sqrt{2} \tilde{E}^{[0 i]} \otimes\left[\tilde{E}^{[j k]}, \alpha(X)\right]-\sqrt{2} \tilde{E}^{[j k]} \otimes\left[\tilde{E}^{[0 i]}, \alpha(X)\right] ; \\
\partial^{*}(\kappa) & =E^{i} \otimes\left[E^{[j k]}, X\right]-E^{[j k]} \otimes\left[E^{i}, \alpha(X)\right] ; \\
\phi \partial^{*}(\kappa) & =\sqrt{2} \tilde{E}^{[0 i]} \otimes\left[\alpha\left(E^{[j k]}\right), \alpha(X)\right]-\tilde{E}^{[j k]} \otimes\left[\alpha\left(E^{i}\right), \alpha(X)\right] \\
& =\sqrt{2} \tilde{E}^{[0 i]} \otimes\left[\tilde{E}^{[j k]}, \alpha(X)\right]-\frac{1}{\sqrt{2}} \tilde{E}^{[j k]} \otimes\left[\tilde{E}_{0}^{i}, \alpha(X)\right] \\
& -\frac{1}{\sqrt{2}} \tilde{E}^{[j k]} \otimes\left[\tilde{E}^{[0 i]}, \alpha(X)\right] .
\end{aligned}
$$

Thus, we get:

$$
\left[\partial^{*}, \phi\right]: E^{i} \wedge E^{[j k]} \otimes X \mapsto-\frac{1}{\sqrt{2}} \tilde{E}^{[j k]} \otimes\left[\Delta \tilde{E}^{i}, \alpha(X)\right] .
$$

Finally, for $\kappa=E^{i} \wedge E^{j} \otimes X, X \in \mathfrak{g}$ we have:

$$
\begin{aligned}
\phi(\kappa) & =2 \tilde{E}^{[0 i]} \wedge \tilde{E}^{[0 j]} \otimes \alpha(X) ; \\
\partial^{*} \phi(\kappa) & =2 \tilde{E}^{[0 i]} \otimes\left[\tilde{E}^{[0 j]}, \alpha(X)\right]-2 \tilde{E}^{[0 j]} \otimes\left[\tilde{E}^{[0 i]}, \alpha(X)\right] ; \\
\partial^{*} \kappa & =E^{i} \otimes\left[E^{j}, X\right]-E^{j} \otimes\left[E^{i}, X\right]-E^{[i j]} \otimes X ; \\
\phi \partial^{*}(\kappa) & =\tilde{E}^{[0 i]} \otimes\left[\tilde{E}^{[0 j]}+\tilde{E}_{0}^{j}, \alpha(X)\right] \\
& -\tilde{E}^{[0 j]} \otimes\left[\tilde{E}^{[0 i]}+\tilde{E}_{0}^{i}, \alpha(X)\right]-\tilde{E}^{[i j]} \otimes \alpha(X) .
\end{aligned}
$$

Thus, we get:

(11) $\left[\partial^{*}, \phi\right]: E^{i} \wedge E^{j} \otimes X \mapsto$

$$
\tilde{E}^{[i j]} \otimes \alpha(X)+\tilde{E}^{[0 i]} \otimes\left[\Delta \tilde{E}^{j}, \alpha(X)\right]-\tilde{E}^{[0 j]} \otimes\left[\Delta \tilde{E}^{i}, \alpha(X)\right] .
$$

Lemma 2. The kernel of the mapping

$$
\left[\partial^{*}, \phi\right]: \wedge^{2} \mathfrak{p}_{+} \otimes \mathfrak{g} \rightarrow \wedge^{2} \tilde{\mathfrak{p}}_{+} \otimes \tilde{\mathfrak{g}}
$$

contains the following spaces:

(a) $\wedge^{2} \mathfrak{g}_{2} \otimes \mathfrak{g}$

(b) $E^{i} \wedge E^{[j k]} \otimes \mathfrak{h}_{i}, i=1, \ldots, l$, where $\mathfrak{h}_{i}$ is a subalgebra in $\mathfrak{g}$ is given by $\mathfrak{h}_{i}=\left\{X \in \mathfrak{g} \mid\left[\Delta \tilde{E}^{i}, \alpha(X)\right]=0\right\}$.

In particular, $\cap_{i=1}^{l} \mathfrak{h}_{i}=\mathfrak{g}_{1}+\mathfrak{g}_{2}$, and the kernel of $\left[\partial^{*}, \phi\right]$ contains $\mathfrak{g}_{1} \otimes \mathfrak{g}_{2} \otimes\left(\mathfrak{g}_{1}+\mathfrak{g}_{2}\right)$. Moreover, the intersection of the kernel with $\wedge^{2} \mathfrak{g}_{1} \otimes \mathfrak{g}$ is trivial. 
Proof. Item (a) follows immediately from (9). To prove the rest we need to show that the images of $\mathfrak{g}_{1} \otimes \mathfrak{g}_{2} \otimes \mathfrak{g}$ and $\wedge^{2} \mathfrak{g}_{1} \otimes \mathfrak{g}$ under the map $\left[\partial^{*}, \phi\right]$ do not intersect.

Let $s: \tilde{\mathfrak{g}} \rightarrow \tilde{\mathfrak{g}}$ be a non-trivial second order automorphism that stabilizes $\alpha(\mathfrak{g})$. Then it is easy to see that $\alpha(\mathfrak{g})$ coincides with the +1 eigenspace $\tilde{\mathfrak{g}}^{1}(s)$ of $s$ in $\tilde{\mathfrak{g}}$, while the elements $\Delta \tilde{E}^{i}$ lie in $\tilde{\mathfrak{g}}^{-1}(s)$. Hence, for any element $X \in \mathfrak{g}$ the bracket $\left[\Delta \tilde{E}^{i}, \alpha(X)\right]$ lies in $\tilde{\mathfrak{g}}^{-1}(s)$ and is either 0 or is linearly independent of $\alpha(Y)$ of any non-zero $Y \in \mathfrak{g}$. According to equations (10) and (11), we see that any non-zero elements in the images of $\mathfrak{g}_{1} \otimes \mathfrak{g}_{2} \otimes \mathfrak{g}$ and $\wedge^{2} \mathfrak{g}_{1} \otimes \mathfrak{g}$ under the map $\left[\partial^{*}, \phi\right]$ are indeed linearly independent.

In particular, the intersection of the kernel of $\left[\partial^{*}, \phi\right]$ with $\wedge^{2} \mathfrak{g}_{1} \otimes \mathfrak{g}$ is trivial. And the intersection of this kernel with $\mathfrak{g}_{1} \otimes \mathfrak{g}_{2} \otimes \mathfrak{g}$ is described by item (b).

Let us decompose $\kappa$ as $\kappa_{1,1}+\kappa_{1,2}+\kappa_{2,2}$, where $\kappa_{i, j} \in \mathfrak{g}_{i} \wedge \mathfrak{g}_{j} \otimes \mathfrak{g}$. Note that this decomposition is $G_{0}$-invariant, but not, in general, $P$-invariant.

Up to now we have not used the fact that $\kappa$ is coclosed and is concentrated in the positive degree of the space $\operatorname{Hom}\left(\wedge^{2}\left(\mathfrak{g}_{-}\right), \mathfrak{g}\right)$. Using these additional facts, we arrive at the following result.

Theorem 5. The extension of $B_{l}$-geometry to $D_{l+1}$-geometry is normal if and only if $\kappa_{1,1}$ vanishes identically.

Proof. According to Lemma 2 the condition $\left[\partial^{*}, \phi\right](\kappa)=0$ implies that $\kappa_{1,1}=0$. Let us prove the converse.

The assertion (a) of Lemma 2 implies $\left[\partial^{*}, \phi\right]\left(\kappa_{2,2}\right)=0$, and it remains to prove that $\left[\partial^{*}, \phi\right]\left(\kappa_{1,2}\right)=0$.

Let us decompose $\kappa=\sum_{i>0} \kappa^{i}$ according to the homogeneity. In particular, we have the decomposition $\kappa_{1,2}=\sum_{i>0} \kappa_{1,2}^{i}$.

The Bianchi identity can be written as:

$$
\partial \kappa(X, Y, Z)=\{\kappa(\kappa(X, Y), Z)\}-\left\{\left(L_{Z^{*}}\right) \kappa(X, Y)\right\}
$$

where the bracket $\{$,$\} denotes the complete anti-symmetrization by X, Y, Z$. Applying this formula to the case $X, Y, Z \in \mathfrak{g}_{-1}$, we immediately see that the righthand side vanishes identically due to the assumption $\kappa_{1,1}=0$. Thus, $\partial \kappa$ vanishes 
identically on $\wedge^{3} \mathfrak{g}_{-1}$. In more detail, for $X, Y, Z \in \mathfrak{g}_{-1}$ we have:

$$
\partial \kappa(X, Y, Z)=\{\kappa([X, Y], Z)\}-\{[X, \kappa(Y, Z)]\}=\left\{\kappa_{1,2}([X, Y], Z)\right\}=0 .
$$

Note that the map $X \wedge Y \mapsto[X, Y]$ establishes an isomorphism of $\wedge^{2} \mathfrak{g}_{-1}$ and $\mathfrak{g}_{-2}$. Then equation (12) means that the complete anti-symmetrization of $\kappa_{1,2}$ interpreted as an element of $\mathfrak{g}_{1} \otimes \wedge^{2} \mathfrak{g}_{1} \otimes \mathfrak{g}$ is identically 0 .

Next, consider the normality condition $\partial^{*} \kappa=0$ in more detail. Denote by $\mathrm{pr}_{1}$ the projection of $\mathfrak{p}_{+} \otimes \mathfrak{g}$ to $\mathfrak{g}_{1} \otimes \mathfrak{g}$ along $\mathfrak{g}_{2} \otimes \mathfrak{g}$. Note that $\operatorname{pr}_{1} \partial^{*}\left(\kappa_{2,2}\right)=0$. Since $\kappa_{1,1}=0$ by assumption, the equation $\partial^{*} \kappa=0$ implies that $\operatorname{pr}_{1} \partial^{*}\left(\kappa_{1,2}\right)=0$. In more detail, $\operatorname{pr}_{1} \partial^{*}$ restricted to $\mathfrak{g}_{1} \otimes \mathfrak{g}_{2} \otimes \mathfrak{g}$ and applied to $\kappa$ can be written as:

$$
\operatorname{pr}_{1} \partial^{*}: \mathfrak{g}_{1} \otimes \mathfrak{g}_{2} \otimes \mathfrak{g} \rightarrow \mathfrak{g}_{1} \otimes \mathfrak{g}, \quad X \otimes Y \otimes Z \mapsto X \otimes[Y, Z],
$$

where $X \in \mathfrak{g}_{1}, Y \in \mathfrak{g}_{2}, Z \in \mathfrak{g}$.

Now, let us prove $\left[\partial^{*}, \phi\right]\left(\kappa_{1,2}^{i}\right)=0$ for each $i$. As $\kappa_{1,2}^{i}$ takes values in $\mathfrak{g}_{i-3}$, according to Lemma 2 this equation is satisfied automatically for $i>3$. It remains to consider the cases $i=1,2,3$.

For $i=1$ we have $\kappa_{1,2}^{1} \in \mathfrak{g}_{1} \otimes \mathfrak{g}_{2} \otimes \mathfrak{g}_{-2}$, and it is the only harmonic part of the curvature. As the space of harmonic curvatures coincides with the traceless part of $\mathfrak{g}_{1} \otimes \mathfrak{g}_{2} \otimes \mathfrak{g}_{-2}$, we derive that all traces of $\kappa_{1,2}^{1}$ should vanish.

We can explicitly write $\kappa_{1,2}^{1}$ as $P_{i[j k]}^{[r s]} E^{i} \otimes E^{[j k]} \otimes E_{[r s]}$. Then according to (10) we have:

$$
\left[\partial^{*}, \phi\right]\left(\kappa_{1,2}^{1}\right)=-\frac{1}{\sqrt{2}} P_{i[j k]}^{[r s]} \tilde{E}^{[j k]} \otimes\left[\Delta \tilde{E}^{i}, \alpha\left(E_{[r s]}\right)\right]=\sqrt{2} \sum_{i} P_{i[j k]}^{[i s]} \tilde{E}^{[j k]} \otimes \tilde{E}_{s} .
$$

As the tensor $P$ is totally traceless (this can also be derived directly from (12) and (13)), we see that $\sum_{i} P_{i[j k]}^{[i s]}=0$ and, thus, $\left[\partial^{*}, \phi\right]\left(\kappa_{1,2}^{1}\right)=0$.

Next, for $i=2$ we have $\kappa_{1,2}^{2}=S_{i[j k]}^{l} E^{i} \otimes E^{[j k]} \otimes E_{l}$. Then according to (10):

$$
\left[\partial^{*}, \phi\right]\left(\kappa_{1,2}^{2}\right)=-\frac{1}{\sqrt{2}} S_{i[j k]}^{l} \tilde{E}^{[j k]} \otimes\left[\Delta \tilde{E}^{i}, \alpha\left(E_{l}\right)\right]=\frac{1}{\sqrt{2}} \sum_{i} S_{i[j k]}^{i} \tilde{E}^{[j k]} \otimes \tilde{E}_{0}^{0} .
$$

But equation (12) implies that $S_{[i j k]}^{l}=0$, and the condition $\operatorname{pr}_{1} \partial^{*}\left(\kappa_{1,2}^{2}\right)=0$ is rewritten as:

$$
S_{i[j k]}^{l} E^{i} \otimes\left[E^{[j k]}, E_{l}\right]=-\sum_{j} S_{i[j k]}^{j} E^{i} \otimes E^{k}=0 .
$$


Hence, $\sum_{j} S_{i[j k]}^{j}=0$ and together with $S_{[i j k]}^{l}=0$ it implies that $\sum_{i} S_{i[j k]}^{i}=0$. Thus, $\left[\partial^{*}, \phi\right]\left(\kappa_{1,2}^{2}\right)=0$ as well.

Similarly, for $i=3$ we have $\kappa_{1,2}^{3}=Z_{i[j k] r}^{s} E^{i} \otimes E^{[j k]} \otimes E_{s}^{r}$. Then according to $(10)$

$$
\left[\partial^{*}, \phi\right]\left(\kappa_{1,2}^{3}\right)=-\frac{1}{\sqrt{2}} Z_{i[j k] r}^{s} \tilde{E}^{[j k]} \otimes\left[\Delta \tilde{E}^{i}, \alpha\left(E_{s}^{r}\right)\right]=-\frac{1}{\sqrt{2}} \sum_{i} Z_{i[j k] r}^{i} \tilde{E}^{[j k]} \otimes \tilde{E}^{r} .
$$

As above, equation (12) implies that $Z_{[i j k] r}^{s}=0$, and the condition $\operatorname{pr}_{1} \partial^{*}\left(\kappa_{1,2}^{3}\right)=$ 0 is rewritten as:

$$
Z_{i[j k] r}^{s} E^{i} \otimes\left[E^{[j k]}, E_{s}^{r}\right]=-\sum_{j} Z_{i[j k] r}^{j} E^{i} \otimes E^{[r k]}=0 .
$$

Hence, $\sum_{j} Z_{i[j k] r}^{j}=0$ and together with $Z_{[i j k] r}^{s}=0$ it implies that $\sum_{i} Z_{i[j k] r}^{i}=0$. Thus, $\left[\partial^{*}, \phi\right]\left(\kappa_{1,2}^{2}\right)=0$.

This completes the proof of the theorem.

Geometrically, the condition $\kappa_{1,1}=0$ from Theorem 5 means that the curvature tensor of the normal Cartan connection associated with the non-degenerate distribution $D$ vanishes identically on $\wedge^{2} D$. It is clear that this condition is well-defined and defines a certain subclass in the class of all non-degenerate $l$ dimensional distributions on $l(l+1) / 2$-dimensional manifolds. The example from S. Armstrong [1] shows that this subclass is not empty.

Example 3 ([1]). Let $l$ be any integer greater or equal to 4 . Let $\left\{x_{i}, y_{[j k]}\right\}$ be a local coordinate system on $M$, where $1 \leq i, j, k \leq l, j<k$. We also define functions $y_{[k j]}$ as $-y_{[j k]}$ for $k>j$. Define a frame:

$$
Y_{[j k]}=\frac{\partial}{\partial y_{[j k]}}, \quad X_{i}=\frac{\partial}{\partial x_{i}}-\sum_{p=i+1}^{l} x_{p} Y_{[i p]} .
$$

$1 \leq j<k \leq l, 1 \leq 1 \leq l$. As before, we define also $Y_{[k j]}=-Y_{[j k]}$ for $k>j$ and $Y_{[j j]}=0$ for any $1 \leq j \leq l$.

Clearly, we have $\left[X_{i}, X_{j}\right]=Y_{[i j]}$ and $\left[X_{i}, Y_{[j k]}\right]=0$ for all $1 \leq i, j, k \leq l$ The distribution $D$ spanned by $X_{1}, \ldots, X_{l}$ defines a flat normal Cartan connection of type $B_{l}$ and has a maximal possible symmetry algebra of dimension $l(l+1) / 2$ among all non-degenerate distributions of rank $l$. 
Now let $X_{1}^{\prime}=X_{1}+y_{[12]} Y_{[34]}$ and $X_{i}^{\prime}=X_{i}$ for $i \geq 2$. Define $D^{\prime}$ as a span of $X_{1}^{\prime}, X_{2}^{\prime}, \ldots, X_{l}^{\prime}$. Is is easy to see that we still have the commutation relation $\left[X_{i}^{\prime}, X_{j}^{\prime}\right]=-Y_{[i j]}$ for all $1 \leq i, j \leq l$. But now we also get an additional non-trivial relation $\left[X_{1}^{\prime}, Y_{[12]}\right]=-Y_{[34]}$.

Thus, using structure equations (2), we see that the only non-vanishing structure coefficient is $f_{1[12]}^{[34]}=1$. Note that the tensor $P$ defined by (5) with $A=0$ is already trace-free. So, we see that functions $A_{j k}^{i}$ and $C_{[j k]}^{i}$ vanish in this case, and the only non-vanishing coefficient of the tensor $P$ is $P_{1[12]}^{[34]}=1$. Proceeding to the coefficients of degree 2 , in the same way we get $E=F=0$ and all curvature parts of degree 2 (tensors $R, S$ and $T$ ) vanish identically. Thus, we get $\omega_{j}^{i}=0$ and $\omega_{i}=0 \bmod D^{\perp}$.

In fact, assuming that $\omega_{i}=0$ and $\omega_{[i j]}=0$ (on $M$ ), we get a $\mathfrak{g}$-valued 1-form on $M$, whose curvature is concentrated in degree 1 . We can always extend this $1-$ form in a unique way to a well-defined Cartan connection $\omega$ on the direct product $\mathcal{G}=M \times P$.

In particular, the structure function $\kappa$ of the constructed normal connection satisfies the condition $\kappa_{1,1}=0$, and the connection itself extends to a normal almost spinorial Cartan geometry. On the other hand, $\kappa \neq 0$, and the distribution $D^{\prime}$ is not equivalent to the model distribution $D$. 


\section{REFERENCES}

[1] S. Armstrong, Free 3-distributions: holonomy, Fefferman constructions and dual distributions, arXiv:0708.3027v3.

[2] R. Bryant, Conformal geometry and 3-plane fields on 6-manifolds, Proceedings of the RIMS symposium "Developments of Cartan geometry and related mathematical problems" (24-27 October 2005).

[3] A. Čap, Correspondence spaces and twistor spaces for parabolic geometries, J. Reine Angew. Math. 582 (2005), 143-172.

[4] A. Čap, H. Schichl, Parabolic Geometries and Canonical Cartan Connections, Hokkaido Math. J., 29, no. 3 (2000), 453-505.

[5] A. Čap, J. Slovák, Parabolic Geometries I: Background and General Theory, Mathematical Surveys and Monographs 164, AMS Publishing House, 2009, 628pp.

[6] É. Cartan, Les systèmes de Pfaff a cinq variables et les équations aux dérivées partielles du second ordre, Ann. Sc. Norm. Sup., 27 (1910), 109-192.

[7] D. Fox, Contact projective structures, Indiana Univ. Math. J., 54 (2005), 1547-1598.

[8] B. Kostant, Lie algebra cohomology and the generalized Borel-Weil theorem, Ann. of Math., 74(1961), 329-397.

[9] P. Nurowski, Differential equations and conformal structures, J. Geom. Phys., 55 (2005), 19-49.

[10] A. Onishchik, On compact Lie groups transitive on certain manifolds, Sov. Math., Dokl. 1 (1961), 1288-1291; translation from Dokl. Akad. Nauk SSSR, 135 (1961), 531-534.

[11] A. Onishchik, Topology of transitive transformation groups, Leipzig: Johann Ambrosius Barth, 1994.

[12] J. Šlhan, Algorithmic computations of Lie algebra cohomologies, Proceedings of the Winter School on Geometry and Physics, Srni 2002, Suppl. Rendiconti Circolo Mat. Palermo, Serie II, 2003, 191-197. http://bart.math.muni.cz/ ${ }^{\sim}$ silhan/lie/

[13] N. Tanaka, On the equivalence problem associated with simple graded Lie algebras, Hokkaido Math. J., 8 (1979), 23-84.

[14] J. Tits, Espaces homogénes complexes compacts, Comment. Math. Helv., 37 (1962), 111120.

[15] K. Yamaguchi, Differential systems associated with simple graded Lie algebras, Advanced Studies in Pure Mathematics, 22 (1993), 413-494.

Boris Doubrov

Department of Applied Mathematics

Belorussian State University, Skoriny av. 4,

220030 Minsk, Belarus

E-mail: doubrov@islc.org

Jan Slovák

Department of Mathematics and Statistics 
Masaryk University, Kotlářská 2a,

61137 Brno, Czech Republic

E-mail: slovak@math.muni.cz 